\section{Waffle-iron filters with oblique boss patterns}

\author{
T Stander ${ }^{1}$ P Meyer $^{1}$ PW van der Walt ${ }^{2}$ \\ W Steyn ${ }^{2}$
}

\begin{abstract}
A waffle-iron filter with an oblique boss pattern is proposed. A circuit model based synthesis method is developed to achieve control over the in-band reflection response. For equivalent filter orders, the boss pattern is found to be more compact than traditional waffle-iron filters
\end{abstract}

\section{INTRODUCTION}

Waffle-iron low-pass filters were first invented by S.B. Cohn et al at the Stanford Research Institute in 1957 [1] and have since become a popular solution for harmonic suppression. This due to the wide stopband, typically covering up to the four times the cutoff frequency [2]. The classical structure, as shown in Fig. 1, consists of uniform multi-ridged rectangular waveguide with equal width transverse grooves. This forms rows and columns of rectangular bosses or pegs in a reduced height waveguide floor and ceiling, matched to standard height waveguide with stepped E-plane sections.

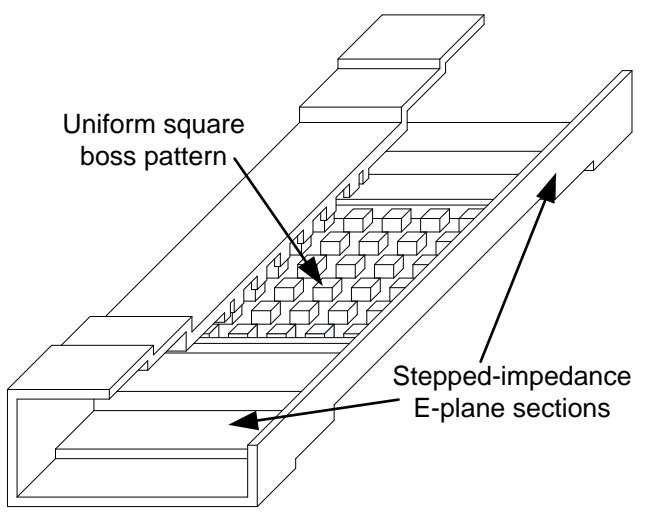

Figure 1: Sectioned view of classical uniform boss waffle-iron filter

The main drawback to these filters is that both classical synthesis methods, outlined in [3], are based on image impedance methods. This method is applicable only if all longitudinal grooves are assumed uniform, and allows for explicit stop-band synthesis, but no direct control over the pass-band transmission function. Later publications [4]

\footnotetext{
${ }^{1}$ University of Stellenbosch, Stellenbosch, South Africa, email: pmeyer@sun.ac.za

${ }^{2}$ Reutech Radar Systems, Stellenbosch, South Africa: email pwvdwalt@rrs.co.za
}

implement non-uniform boss patterns, but the synthesis requires full-wave optimization to achieve equi-ripple in-band reflection responses. These perturbations are still normal to the incident $\mathrm{z}$ directed $\mathrm{TE}_{\mathrm{m} 0}$ mode. This paper investigates TEM propagation inside the filter cavity, and proposes a non-uniform oblique boss pattern to perturb the TEM phase path. Fast circuit optimization is used to synthesize the boss pattern. In all cases, the circuit models are constructed and simulated in AWR Microwave Office, and compared to a full-wave simulation model in CST Microwave Studio

\section{TEM PROPAGATION}

$\mathrm{TE}_{\mathrm{m} 0}$ mode propagating in rectangular waveguide can be decomposed into two diagonally propagating TEM modes [5] in the guide as shown in Fig. 2. The propagating angle of the diagonal mode $\theta$ is such that the path length from sidewall to sidewall is a multiple of half a free-space wavelength $\lambda_{0}$. By having both transversal and longitudinal corrugating grooves, as for the uniform waffle-iron filter boss pattern shown in Fig. 3, identical uniform perturbations of length $\varphi$ are formed along both TEM propagating phase paths in the special case where $\theta=45^{\circ}$. In this view, it is effectively the TEM mode that is filtered. Since all propagating $\mathrm{TE}_{\mathrm{m} 0}$ modes decompose into the same two diagonal TEM paths at a specific frequency, the filter has similar transmission responses for all incident $\mathrm{TE}_{\mathrm{m} 0}$ modes. $\mathrm{TE}_{0 \mathrm{n}}$ modes may safely be neglected in analysis since the corrugated sections are of reduced waveguide height

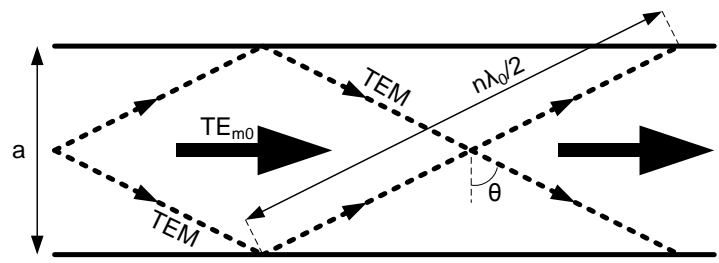

Figure 2: TEM propagation paths in rectangular waveguide

By rotating the bosses by an angle $\theta$ and making them of non-uniform length, as shown in Fig. 4, perforated corrugations are formed with normal incidence to the TEM phase path (but oblique to $\mathrm{TE}_{\mathrm{m} 0}$ propagation). This means that, at multiples of some frequency $\mathrm{f}_{0}=\mathrm{c} / \lambda_{0}$, any incident wave can be presented with a user-defined perturbation pattern. As with classical boss patterns, this approach is restricted to patterns with $\theta=45^{\circ}$ (to ensure normal incidence to both TEM phase paths) and has to be axially symmetric (to avoid dissimilar perturbation paths for the two TEM phase paths). 


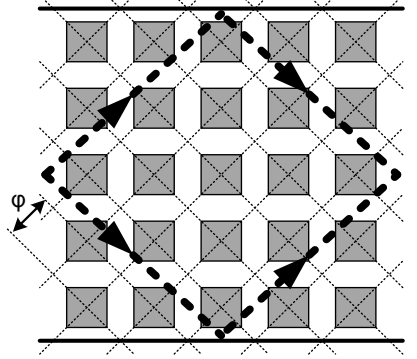

Figure 3: TEM phase path perturbation by uniform boss pattern with oblique angle of incidence.

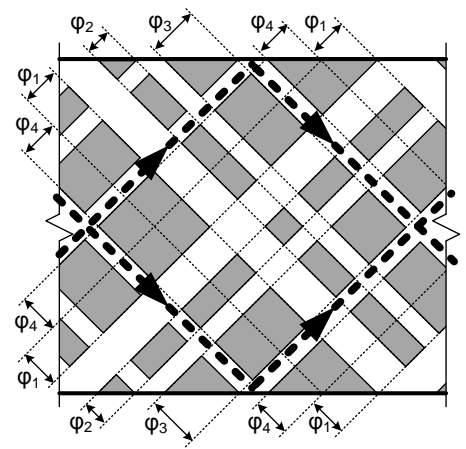

Figure 4: TEM phase path perturbated by nonuniform boss pattern with normal angle of incidence.

\section{DESIGN}

A very simple circuit model can be used for the design of these filters. As they are essentially TEM filters, the Marcuvitz model for shorted-stub-loaded transmission lines are used [3] with all reactive compensation removed to aid circuit optimization. No synthesis method for these non-uniform structures has, as yet, been published, but circuit simulators (as opposed to full-wave simulators) are fast enough to find an optimized response that adheres to the filter requirements.

\section{RESULTS}

The simulated results using the circuit model, a fullwave model directly calculated from the circuit model, and a tuned full-wave model for an oblique waffle-iron filter is shown in Fig. 6. Although some fine tuning is necessary, the initial full-wave model (derived from the optimized circuit model) is established much faster than a model optimized in a full-wave solver. In addition, the filter can be shown to be more compact than normal waffle-iron filters. The spurious resonances excited inside the filter cavity are of some concern, since these are unaccounted for in the circuit model. This aspect has to be evaluated with some scrutiny in any design.
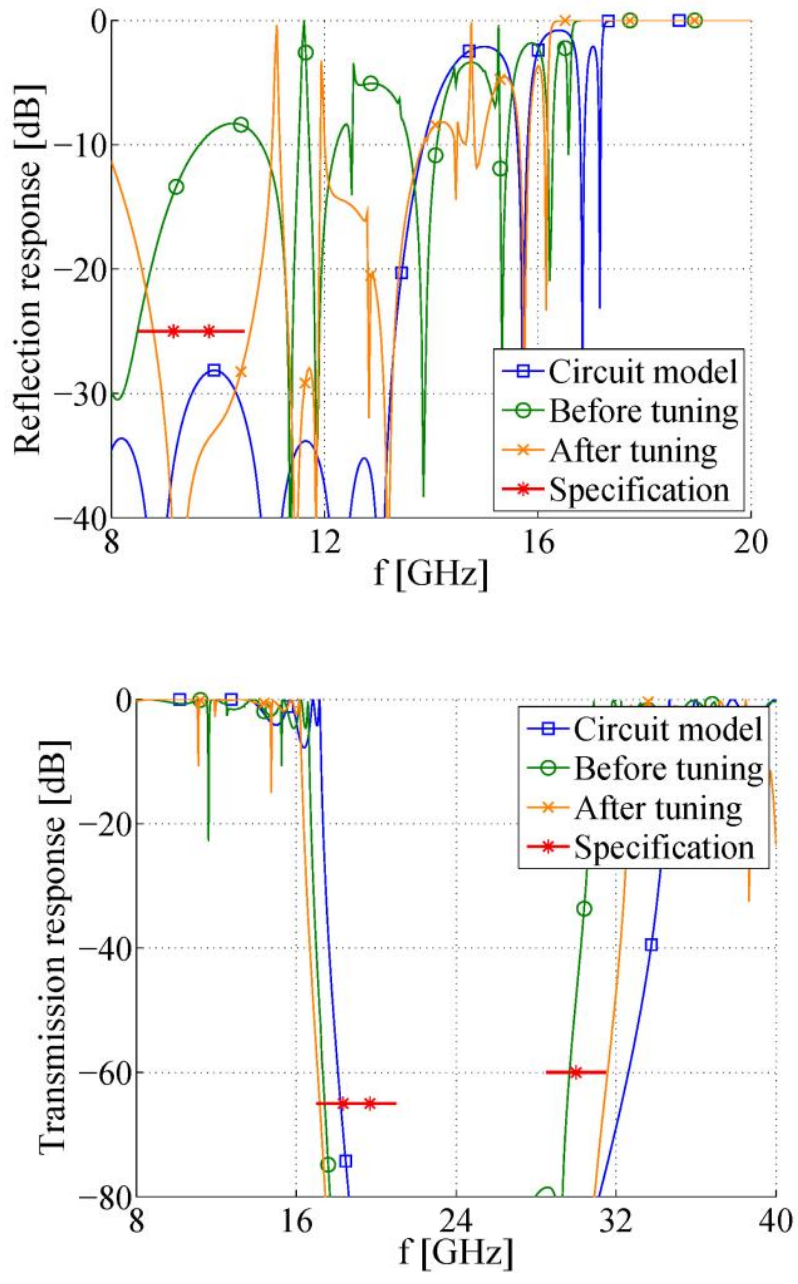

Figure 6: Simulated response of Oblique Waffle-Iron Filter.

\section{References}

[1] S.B. Cohn, E. M.T. Jones, O. Heinz, J.K. Shimizu, B.M. Schiffman, and F.S. Coale, "Research on design criteria for microwave filters," Stanford Research Institute, Final Report - SRI Project 1331, June 1957.

[2] L. Young, "Postscript to two papers on waffleiron filters (correspondence)," IEEE Transactions on Microwave Theory and Techniques, vol. 11, no. 6, pp. 555 - 557, November 1963.

[3] G. L. Matthaei, L. Young, and E. M. T. Jones, Microwave Filters, Impedance-Matching Networks, and Coupling Structures. McGraw-Hill Book Co, 1964.

[4] F. Arndt and J. Brandt, "Direct EM based optimization of advanced waffle-iron and rectangular combline filters," in IEEE MTT-S International Microwave Symposium Digest, June 2002 